\title{
Prospects for Standard Model Measurements at the High-Luminosity LHC with CMS
}

\author{
Ferdos Rezaei Hosseinabadi*† \\ Institute for Research in Fundamental Sciences (IPM), Iran. \\ E-mail: ferdos.rezaei.hosseinabadidcern.ch
}

\begin{abstract}
The prospects for selected standard model measurements at the High-Luminosity LHC are presented. These studies investigate the potential of the upgraded CMS detector at the HL-LHC for precision measurements of the top mass and a flavour changing neutral currents search in single top quark production. An extrapolation to the proton proton collisions at a centre-of-mass energy of $14 \mathrm{TeV}$ with the HL-LHC run conditions is performed assuming an integrated luminosity of up to $3 \mathrm{ab}^{-1}$.
\end{abstract}

EPS-HEP 2017, European Physical Society conference on High Energy Physics 5-12 July 2017

Venice, Italy

${ }^{*}$ Speaker.

${ }^{\dagger}$ on behalf of the CMS Collaboration 


\section{Introduction}

HL-LHC is the proposed upgrade project for the LHC which is going to start its operation by 2023. It will function at a luminosity of $5(7.5) \times 10^{34} \mathrm{~cm}^{2} \mathrm{~s}^{-1}$, corresponding to an average pile up of $\mu=140(200)$. It has a goal of accumulating up to $3 \mathrm{ab}^{-1}$ proton-proton collision data at a centre-of-mass energy of $14 \mathrm{TeV}$. In presence of high luminosity collisions, a radiation tolerant high granularity detector is needed. To maintain and improve the physics performance, coverage of the detector is expected to be extended. These requirements are considered for the phase II upgrade of CMS detector. It is assumed that the CMS tracker will be extended up to $|\eta|=3.8$ and a selective readout of tracks with $p_{T}>2 \mathrm{GeV}$ will be used for triggering purposes. In the upgraded muon system, muon tagging will be possible up to $|\eta|=3$. The detector endcaps will consist of radiation tolerant high granular silicon hadronic and electromagnetic calorimeters with 3-dimensional readout capabilities.

The achievable precision for top quark mass measurements based on different analysis strategies is estimated with the HL-LHC run conditions. Flavour changing neutral current (FCNC) is studied in single top production in association with a photon and expected limits are set on branching fraction of top decays to an "up" quark and a photon. The feasibility of a proposed track trigger for the B physics studies $\left(B_{s} \rightarrow \phi \phi\right)$ is also discussed in [1].

\section{Challenges of upgrade physics studies}

There are various complications in a physics study at HL-LHC. They include the concern for the detector simulation especially in presence of large pile up, as well as estimation of backgrounds contamination and systematic uncertainties. The latter would be more challenging if a background is usually estimated from data for some reasons. Regarding the MC event generation for signal and background processes, CMS has chosen (for most of studies) to use a fast simulation of detector using its simplified description and parametrized response taken from full simulation.

\section{Prospect of Top mass measurement precision at HL-LHC}

Top quark mass is a fundamental parameter of standard model (SM) which is renormalisation scheme dependent. Its precise value provides a key input to the global electroweak fit and leads to a significant constraint on stability of the electroweak vacuum. Higher rate of events at HL-LHC provides the possibility for high precision differential measurements of $\mathrm{m}_{\mathrm{t}}$ and gains insight into more tunes in the dark corners of phase space.

There are several methods for measuring top "MC mass", which are listed below. However in a well-defined renormalisation scheme it can be extracted using dependency of predicted NNLO top quark pair production cross section on top quark "pole mass". Precision in pole mass is not competitive with MC mass, but it provides a more straightforward interpretation of the measurement.

- In the reference method which is the most precise approach, top mass together with a jet energy scale factor are inferred from a fit to the invariant mass of top decay products in semileptonic decays of top pair events. This measurement is suffered mostly from uncertainty on jet energy corrections. 
- A same approach as the reference method can be employed on single top events. This method has advantage of not being very sensitive to the modelling of QCD interactions, because single top is produced in an electroweak process.

- There are also tracking-based methods, for instance by using secondary vertex information of $b$ hadron. This method avoids the full reconstruction of jets, therefore strongly reduces the traditional uncertainties related to the jet energy calibration and QCD modelling. There is also a lower dependency on pileup as charged tracks only are used.

- As another tracking-based method, a partial reconstruction of top quarks in leptonic decay of $J / \psi$ from the b quark fragmentation $\left(J / \psi \rightarrow \mu^{+} \mu^{-}\right)$is used to determine top mass through its correlation with the mass of the $J / \psi+\ell$ system, where $\ell$ is either a muon or an electron produced in the leptonic decay of the accompanying $\mathrm{W}$ boson.

The achievable improvement in precision of these methods are evaluated for HL-LHC. In this document the reference method is discussed and a comprehensive plot is shown comparing various methods. Details of expected precision for other methods can be found at [1].

The reference method at $8 \mathrm{TeV}$ measurement leads to $\mathrm{m}_{\mathrm{t}}=172.35 \pm 0.16$ (stat) \pm 0.48 (syst) $\mathrm{GeV}$, corresponding to a precision better than $0.3 \%$. The main sources of uncertainty are related to the hadronization modelling, the flavor-dependent jet energy scale (JES), the branching fractions of semileptonic $b$ hadron decays, the global JES, and the choice of ME generator.

The prospect of top mass measurement at HL-LHC was studied before based on $7 \mathrm{TeV}$ measurement at CMS [2]. The arguments in that document are used to predict uncertainties at $14 \mathrm{TeV}$ from those at $8 \mathrm{TeV}$, without new simulations. A summary of expected impact of different sources of uncertainty is shown in table 1 . The values have been conservatively symmetrized. In this document, the assumptions used to estimate those numbers are discussed partially.

The uncertainty due to background rate is decreased by a factor of 3 as $t \bar{t}$ cross section from $8 \mathrm{TeV}$ to $14 \mathrm{TeV}$ grows more rapidly than backgrounds. Since the use of NLO MC generator is already established, the uncertainty due to choice of LO MC generator will not be relevant. At the HL-LHC severe pileup conditions are expected, however the "pileup per particle identification (PUPPI)" algorithm is employed to keep its impact under control. It is assumed that the decrease in trigger efficiency due to higher $p_{T}$ threshold will be compensated by the increase in $t \bar{t}$ cross section at 14 $\mathrm{TeV}$. The modelling of QCD processes (color reconnection, fragmentation, underlying event and parton showering, ...) are studied in 8 and $13 \mathrm{TeV}$ measurements and new parameters value and tunes will be used in the next MC production. A differential k-factors for the MC simulation can be achieved by means of differential predictions for $t \bar{t}$ production at NNLO. Using this k-factor and other constraints from 7 and $8 \mathrm{TeV}$ measurements leads to a reduction in the top quark $p_{T}$ uncertainty, roughly by a factor of 6 . For those uncertainties derived from MC samples, higher statistics of samples will help to constrain the uncertainty. At higher collision energy, the parton density functions (PDF) uncertainty benefits from the production at lower gluon momentum fractions, where it is known with less uncertainty.

The predicted total uncertainty in top mass measurement using $3 \mathrm{ab}^{-1}$ data is less than $0.17 \mathrm{GeV}$ (relative precision below $0.1 \%$ ), with a statistical contribution of $0.02 \mathrm{GeV}$. Main sources of un- 
certainty are expected to be the modelling of underlying event and hadronization. Although, they are expected to be highly reduced by the $3 \mathrm{D}$ fit technique, the foreseen improvements in the $t \bar{t}$ modelling and residual energy corrections for $b$ jets.

Figure 1 summarizes the expected uncertainty on $\mathrm{m}_{\mathrm{t}}$ for the different methods. During the Run 1, most analyses were systematic limited except for the $J / \psi$ method which gains a lot from the higher rate of events at HL-LHC.

\begin{tabular}{lccll} 
& \multicolumn{3}{c}{ Value $(\mathrm{GeV})$} & \\
Source & $8 \mathrm{TeV}$, & $14 \mathrm{TeV}$, & $14 \mathrm{TeV}$, & Comment \\
& $19.7 \mathrm{fb}^{-1}$ & $0.3 \mathrm{ab}^{-1}$ & $3 \mathrm{ab}^{-1}$ & \\
\hline \hline Method calibration & \pm 0.04 & \pm 0.02 & \pm 0.02 & MC stat. $\times 4$ \\
Lepton energy scale & +0.01 & \pm 0.01 & \pm 0.01 & unchanged \\
Global JES & \pm 0.13 & \pm 0.12 & \pm 0.04 & 3D fit, differential \\
Flavor-dependent JES & \pm 0.19 & \pm 0.17 & \pm 0.06 & 3D fit, differential \\
Jet energy resolution & -0.03 & \pm 0.02 & $<0.01$ & differential \\
$E_{T}^{\text {miss }}$ scale & +0.04 & \pm 0.04 & \pm 0.04 & unchanged \\
b tagging efficiency & +0.06 & \pm 0.03 & \pm 0.03 & improved with data \\
Pileup & -0.04 & \pm 0.04 & \pm 0.04 & unchanged \\
Backgrounds & +0.03 & \pm 0.01 & \pm 0.01 & cross sections \\
\hline ME generator & $-0.12 \pm 0.08$ & - & - & NLO ME generator \\
Ren. and fact. scales & $-0.09 \pm 0.07$ & \pm 0.06 & \pm 0.06 & NLO ME generator, \\
& & & & MC stat. \\
ME-PS matching & $+0.03 \pm 0.07$ & \pm 0.06 & \pm 0.06 & MC stat. \\
Top quark $p$ & +0.02 & $<0.01$ & $<0.01$ & improved with data \\
b fragmentation & $<0.01$ & $<0.01$ & $<0.01$ & unchanged \\
Semileptonic b hadron decays & -0.16 & \pm 0.11 & \pm 0.06 & improved with data \\
Underlying event & $+0.08 \pm 0.11$ & \pm 0.14 & \pm 0.09 & improved with data, \\
& & & & MC stat. \\
Color reconnection & $+0.01 \pm 0.09$ & \pm 0.05 & $<0.01$ & improved with data \\
PDF & \pm 0.04 & \pm 0.03 & \pm 0.02 & improved with data \\
\hline \hline Systematic uncertainty & \pm 0.48 & \pm 0.30 & \pm 0.17 & \\
Statistical uncertainty & \pm 0.16 & \pm 0.04 & \pm 0.02 & \\
\hline \hline Total & \pm 0.51 & \pm 0.31 & \pm 0.17 & \\
\hline
\end{tabular}

Table 1: Summary of systematic uncertainties on $\mathrm{m}_{\mathrm{t}}$ measurement in lepton+jets channel. Experimental uncertainties are separated from theoretical ones[1].

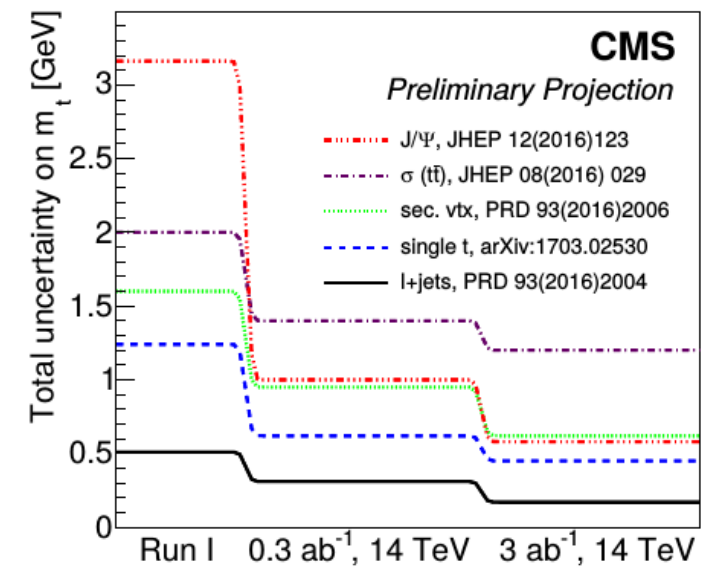

Figure 1: Expected uncertainty on $\mathrm{m}_{\mathrm{t}}$ measurements obtained with projections from RunI measurements to the foreseen condition at HL-LHC[1].

\section{Increase in sensitivity on the search for FCNC single top quark production in association with a photon at HL-LHC}

Top quark FCNC transition are not present at tree level within SM, but can be enhanced in different models beyond the SM. For instance, predicted branching fraction for $t \rightarrow \gamma u$ and $t \rightarrow \gamma c$ decays are approximately $10^{-16}$ and $10^{-14}$, respectively. They can grow several order of magnitudes up to the order of $10^{-6}$ in several models like Minimal Supersymmetric SM and two-higgs doublet model. Therefore, evidence for new physics can be achieved by measuring these branching fractions. Searches in the previous collider experiments as well as LHC experiments found no evidence for FCNC processes. The most stringent constraints on these branching fraction are set by the CMS experiment equal to $B(t \rightarrow u+\gamma)<0.016 \%$ and $B(t \rightarrow c+\gamma)<0.182 \%$ respectively. They are obtained through search for single-top quark production in association with a photon [3].

The expected sensitivity of the upgraded CMS detector at HL-LHC to the $t \rightarrow q \gamma$ FCNC transition is studied using single top and top pair events in the foreseen integrated luminosity. The $t q \gamma$ coupling appears in the production of single top plus photon and in the decay of top quark to a photon and a charm or up quark, in the top pair events. It is assumed that top quark in single top and the second top in top pair events decay leptonically to a bottom quark, a muon and a neutrino. The simulated samples for signal are generated with the MADGRAPH generator interfaced with 

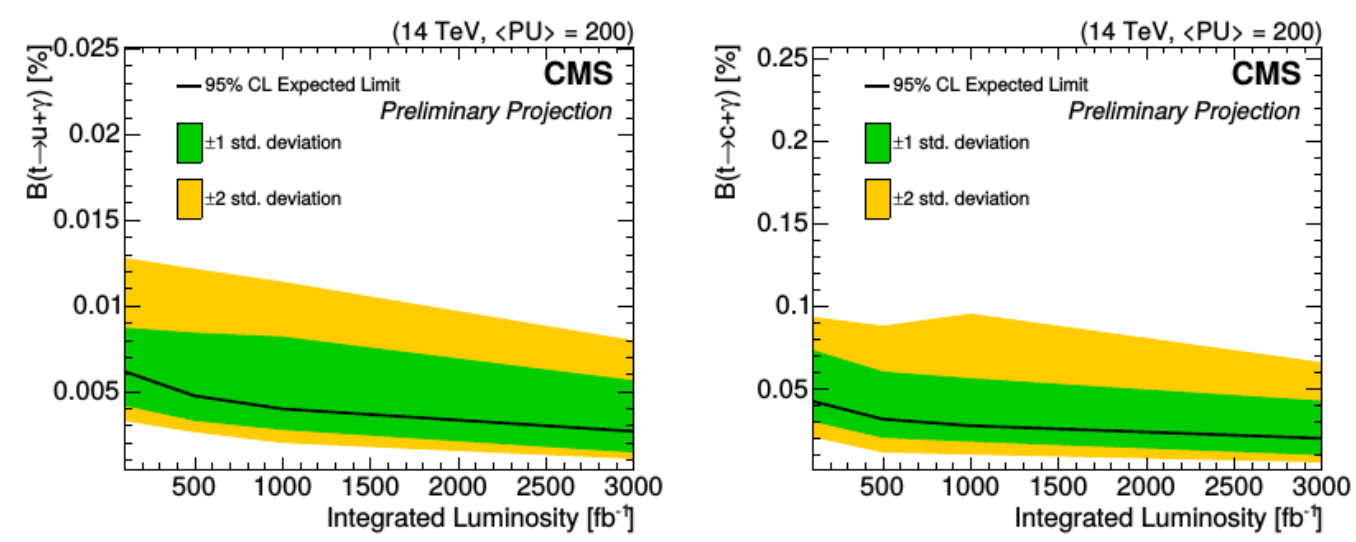

Figure 2: Expected upper limits at $95 \% \mathrm{CL}$ and \pm 1 and \pm 2 standard deviations bands on the branching fractions of $t \rightarrow u+\gamma$ (left) and $t \rightarrow c+\gamma$ (right) as a function of integrated luminosity[1].

PYTHIA for parton showering and hadronization using the NNPDF23 PDFs with the top mass set to $172.5 \mathrm{GeV}$. A fast simulation of the CMS detector is employed to model detector response in presence of 200 additional pileup interactions per bunch crossing. Events are selected requiring presence of exactly one muon $\left(p_{T}>25, \eta<2.5\right)$ passes high purity identification requirements, a jet passes a b-tagging criteria with $70 \%$ efficiency and $1.5(18) \%$ misidentification probability for light (c) jets and a well-identified photon $\left(p_{T}>50, \eta<2.5\right)$ which is well separated from muon and b jet $(\Delta R(\gamma, b)>0.7, \Delta R(\gamma, \mu)>0.7)$. Events are required to have $E_{T}^{\text {miss }}>30 \mathrm{GeV}$. Top quark is reconstructed using four momentum of lepton, neutrino and $\mathrm{b}$ jet and finally events in top mass window $130<m_{t}<220 \mathrm{GeV}$ are studied. The expected efficiencies of $t u \gamma$ and $t c \gamma$ signal processes are found to be $2.30 \%$ and $2.34 \%$, respectively. Although HL-LHC configuration will have photon coverage up to $|\eta|=4$, the selection follows Run I analysis allowing for extrapolation of fake backgrounds $(W+j e t s, t \bar{t}$ and $t+j e t s)$ and systematics from $8 \mathrm{TeV}$ to the HL-LHC conditions. For this study, two scenarios for the systematic uncertainties are considered. In first scenario, the relative total uncertainty is taken from $8 \mathrm{TeV}$ analysis $(11.5 \%$ and $11.0 \%$ for the $t u \gamma$ and $t c \gamma)$ while in the second scenario, theoretical uncertainties are reduced by a factor of two with some improvement for the experimental ones. With the assumption of observing no excess from signal in data, the 95\% CL upper limits on the $B(t \rightarrow u+\gamma)$ and $B(t \rightarrow c+\gamma)$ in the first (second) scenario are set at $4.6 \times 10^{-5}\left(2.7 \times 10^{-5}\right)$ and $3.4 \times 10^{-4}\left(2.0 \times 10^{-4}\right)$, respectively, using an integrated luminosity of $3 a b^{-1}$. Limits are presented in terms of integrated luminosity in Fig. 2 for the second scenario.

\section{References}

[1] CMS Collaboration, ECFA 2016: Prospects for selected standard model measurements with the CMS experiment at the High-Luminosity LHC, CMS-PAS-FTR-16-006 (2017), http: //cds.cern.ch/record/2262606

[2] CMS Collaboration, Projected improvement of the accuracy of top-quark mass measurements at the upgraded LHC, CMS-PAS-FTR-13-017 (2013), http: / / cds . cern. ch/record/1605627

[3] CMS Collaboration, Search for anomalous single top quark production in association with a photon in pp collisions at sqrt(s) = $8 \mathrm{TeV}$, JHEP 04 (2016) 035 , doi:10.1007/JHEP04(2016)035 (2016) 